\title{
Tilted response of fishnet metamaterials at near-infrared optical wavelengths
}

\author{
Alexander Minovich, ${ }^{1}$ Dragomir N. Neshev, ${ }^{1}$ David A. Powell, ${ }^{1}$ Ilya V. Shadrivov, ${ }^{1}$ Mikhail Lapine, ${ }^{1,2}$ Ian McKerracher, ${ }^{3}$ \\ Haroldo T. Hattori, ${ }^{4}$ Hark Hoe Tan, ${ }^{3}$ Chennupati Jagadish, ${ }^{3}$ and Yuri S. Kivshar ${ }^{1}$ \\ ${ }^{1}$ Nonlinear Physics Centre, Research School of Physics and Engineering, The Australian National University, Canberra, \\ Australian Capital Territory 0200, Australia \\ ${ }^{2}$ Department of Electronics and Electromagnetics, Faculty of Physics, University of Seville, Avenida Reina Mercedes s/n, 41012 Sevilla, \\ Spain \\ ${ }^{3}$ Department of Electronic Materials Engineering, Research School of Physics and Engineering, The Australian National University, \\ Canberra, Australian Capital Territory 0200, Australia \\ ${ }^{4}$ School of Engineering and Information Technology, The Australian Defence Force Academy, University of New South Wales, Canberra, \\ Australian Capital Territory 2600, Australia
}

(Received 28 September 2009; revised manuscript received 18 December 2009; published 5 March 2010)

\begin{abstract}
We study experimentally the transmission properties of $\mathrm{Au}-\mathrm{TiO}_{2}-\mathrm{Au}$ fishnet metamaterials in the nearinfrared spectral range and analyze the change in the transmission resonances at varying angles of incidence and different input polarizations. The results show that the main transmission peak through the fishnet is due to the excitation of hole modes. This high-transmission region is significantly influenced by surface plasmon coupling when the incident electric field has a component normal to the metal plates, while little change with respect to tilt is observed when the electric field is parallel to the two metal films of the fishnet.
\end{abstract}

DOI: 10.1103/PhysRevB.81.115109

PACS number(s): 42.25.Bs, 42.25.Fx, 78.67.-n

\section{INTRODUCTION}

The ability to engineer the properties of materials through nanofabrication has driven research into optical metamaterials with a negative refractive index. ${ }^{1}$ Following the experimental demonstrations at microwave frequencies, ${ }^{2}$ continuous attempts are being made to scale metamaterials down to optical wavelengths. One of the most popular designs in that search is the fishnet metal-dielectric-metal structure. ${ }^{3-10}$

At an intuitive level, the behavior of the fishnet structure is usually explained by conceptually dividing the structure into capacitive and inductive components, accountable for the electric and magnetic responses of the material, respectively. ${ }^{5}$ In particular, the magnetic response comes from the out-of-phase currents in the two metal plates, which resembles the response of cut-wire pairs. ${ }^{11}$ An alternative physical picture of the fishnet metamaterial behavior is given by the analysis of the plasmon modes in the structure. . $^{12,13}$ These analyses suggest that the negative refraction in fishnet structures appears due to the interaction of the hole modes in the perforated film with the modes of the dielectric slab sandwiched between the metal layers.

Because of the strong spatial dispersion ${ }^{14}$ in fishnet structures, to date most authors have studied the case of normal incidence in attempts to describe the response in terms of effective parameters. The magnetic response of fishnets at different inclination angles was first studied in Ref. 4, where the authors observed a dip in the transmission spectrum, which they attributed to the excitation of magneto-inductive waves propagating along the structures. This magnetic plasmon resonance experiences a blue shift with an increase in the angle of incidence for both orthogonal input polarizations. ${ }^{4}$ Importantly, such magnetic plasmons are associated with current circulations in the structure and the appearance of induced magnetic moments. ${ }^{15}$ Subsequent studies ${ }^{16,17}$ have shown that different magnetic plasmon reso- nances with complex dispersion spectra can be excited in the fishnet structures.

In this work, we study experimentally and numerically the transmission through a fishnet structure for different inclination angles and different incident polarizations. We analyze the origin of the transmission peaks in our fishnet structure and show that the underlying modes are not necessarily linked to excitation of magnetic plasmons but rather they are associated with the phenomenon of extraordinary optical transmission through the subwavelength holes. By monitoring the electric field distribution, we obtain the current flow on the surface of the two metal films and show that they are qualitatively similar to those predicted for negative-index regime of the fishnet. ${ }^{18}$ The fields in the top and bottom metal films, however appear in-phase. This indicates that the effective magnetic response in the structure is negligible at the wavelength of maximum transmission.

Furthermore, we study the angular dispersion of the fishnet structure in the near-infrared spectral region and we observe a red shift of the main transmission maximum with sample inclination when the incident polarization has an electric field component normal to the metal films. For the orthogonal polarization, parallel to the metal films, the response of the structure does not depend on the angle of incidence. These findings give guidelines for selecting the inclination of fishnet slabs $^{19}$ and wedges, ${ }^{9}$ with possible application to beam steering through metamaterials.

In Sec. II, we describe the fabrication of our structure and the arrangement of our measurement system. In Sec. III, we describe the measured and simulation results for normally incident waves. Finally in Sec. IV, we show the response of the structure to a beam incident at an oblique angle.

\section{EXPERIMENTAL SETUP}

Our sample consists of a $\mathrm{Au}-\mathrm{TiO}_{2}$-Au metal-dielectricmetal structure. The layers were deposited on a quartz sub- 


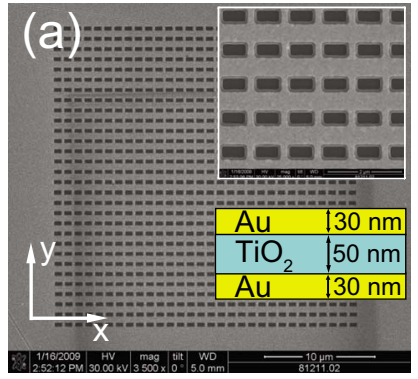

(b)

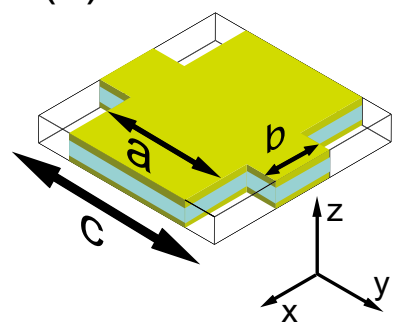

(c)
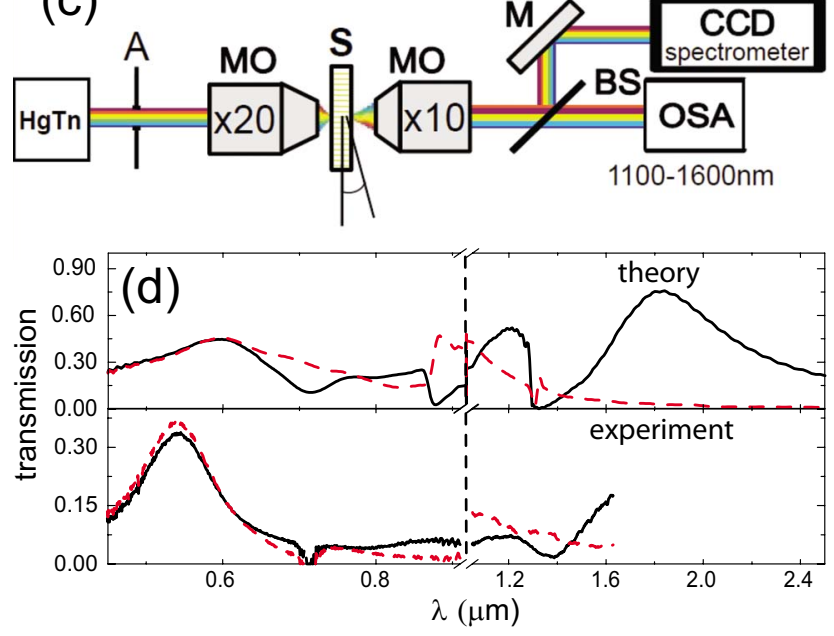

FIG. 1. (Color online) (a) Fishnet metamaterial structure consisting of 30-50-30 nm Au- $\mathrm{TiO}_{2}-\mathrm{Au}$ films. (b) Unit cell with $a$ $=565 \mathrm{~nm}, b=265 \mathrm{~nm}$, and $c=860 \mathrm{~nm}$. (c) Experimental setup: HgTn-150 W halogen white light source, A-aperture, MOmicroscope objective, $\mathrm{S}$ - sample with tilting, BS-beam splitter, $\mathrm{M}$-mirror, OSA - optical spectrum analyzer. (d) Calculated (top) and experimentally measured (bottom) metamaterial transmission spectra for normal incidence. The main transmission peak is in the spectral range 1.5-2.2 $\mu \mathrm{m}$. Solid line-incident electric field $E \| y$, dashed line-incident electric field $E \| x$.

strate. DC magnetron sputtering in an Ar atmosphere was used to deposit the gold films, while radio frequency (RF) magnetron sputtering was used for the deposition of the $\mathrm{TiO}_{2}$ dielectric. The refractive index of the $\mathrm{TiO}_{2}$ film was measured by a white-light ellipsometer, yielding a refractive index in the form $n(\lambda)=2.2744+0.030759 / \lambda^{2}$ $+0.0037163 / \lambda^{4}$. The three layers were patterned by a focused ion beam (FIB) FEI XP200 milling system to form rectangular holes of $595 \times 295 \mathrm{~nm}$ size, arranged in a square lattice with a period of $860 \mathrm{~nm}$. A scanning electron microscope (SEM) image and structural parameters of the sample are shown in Figs. 1(a) and 1(b).

Due to the small sample size of $20 \times 20 \mu \mathrm{m}$, we have developed a microspectrometer system to characterize the transmission through the sample [see Fig. 1(c)]. A small aperture $\mathrm{A}$ is illuminated by a $100 \mathrm{~W}$ halogen-tungsten white source and its demagnified image is projected onto the sample. The transmitted light is then collected by a collimating microscope objective. We use a $20 \times$ achromatic microscope objective lens with a numerical aperture $(\mathrm{NA})=0.45$ for illumination and a $10 \times$ objective $(\mathrm{NA}=0.20)$ for colli- mation. The output transmission in the spectral range $1.0-1.7 \mu \mathrm{m}$ is recorded by an optical spectrum analyzer, while the transmission in the spectral range $0.45-0.9 \mu \mathrm{m}$ is measured by a charge-coupled device spectrometer. In both cases, the transmission is normalized to the transmission of an uncoated substrate.

\section{TRANSMISSION SPECTRA AND MODES OF FISHNET METAMATERIALS}

The experimentally obtained transmission spectrum for normal incidence onto the sample is shown in Fig. 1(d), bottom. Two incident polarizations were measured $-E \| x$ and $E \| y$ — shown with the dashed and solid curves, respectively. For both input polarizations, the measured spectrum shows a transmission peak at $\sim 0.5 \mu \mathrm{m}$, which is also observed in the response of the unpatterned gold layer. A complex pattern of resonances is observed in the intermediate spectral range 0.7-1.2 $\mu \mathrm{m}$. Above $1.5 \mu \mathrm{m}$ the transmission shows strong polarization anisotropy, where the incident beam with electric field polarized along the thin wires of the fishnet $(E \| y)$ experiences a transmission peak, while the orthogonally polarized incident field $(E \| x)$ shows a drop in transmission. Such a strong anisotropy in transmission is typically observed in negative index fishnet structures. ${ }^{5}$ Unfortunately, due to the limited spectral range of our detection system, we are not able to measure the transmission band at wavelengths beyond $1.7 \mu \mathrm{m}$. Therefore, we resort to numerical simulations in order to uncover the whole spectral range of interest. For the simulations we use a finite difference time domain (FDTD) method performed with commercial software (RSOFT). In our simulations, we neglected the dispersion of the dielectrics, using constant refractive indices of 1.5 for the quartz substrate and 2.7 for the $\mathrm{TiO}_{2}$. This approximation can be justified since the refractive index of the $\mathrm{TiO}_{2}$ layer changes only $7 \%$ in the spectral range of 500 to $2400 \mathrm{~nm}$ [the range of Fig. 1(d)]. This is a negligible change in comparison to the dispersion of the gold films. For the dielectric permittivity of gold we use six-term fit expression from the RSOFT material library

$$
\varepsilon(\omega)=\varepsilon_{\infty}+\sum_{k} \frac{\Delta \varepsilon_{k}}{-\omega^{2}-b_{k}(i \omega)+c_{k}},
$$

where $\varepsilon_{\infty}=1, \omega$ is the angular frequency in $\mu \mathrm{m}^{-1}$ (in the RSOFT system of units time $T=c t[\mu \mathrm{m}]$ ) and the values of $\Delta \varepsilon_{k}, b_{k}$, and $c_{k}$ are given in Table I. ${ }^{20}$

In the simulations we use a nonuniform spatial grid with size varying from $2 \mathrm{~nm}$ near the metal-dielectric interfaces to $10 \mathrm{~nm}$ in free space. Time step $(c t)$ is $8 \times 10^{-4} \mu \mathrm{m}$. For calculation of the transmission we excite the system by a short chirped pulse with a broad spectrum and plane wave front. The numerically calculated spectra are shown in Fig. 1(d), top. We find good qualitative agreement between the experimentally measured and numerically calculated spectra. In the simulations, we observe a high-transmission resonance around $1.8 \mu \mathrm{m}$, when the incident electric field is aligned along the thinner metal strips of the metamaterial $(E \| y)$. This peak is absent for the other polarization $(E \| x)$. 
TABLE I. Coefficients for the dispersion model of gold (Ref. 20).

\begin{tabular}{lcccccc}
\hline \hline$k$ & 1 & 2 & 3 & 4 & 5 & 6 \\
\hline$\Delta \epsilon_{k}$ & 1589.52 & 50.1953 & 20.9147 & 148.494 & 1256.97 & 9169 \\
$b_{k}$ & 0.26842 & 1.22055 & 1.74726 & 4.40613 & 12.63 & 11.2128 \\
$c_{k}$ & 0 & 4.41746 & 17.6698 & 226.098 & 475.139 & 4550.76 \\
\hline \hline
\end{tabular}

We also study the possibility to control the position of transmission peaks by scaling the geometry of the sample. We find that the transmission peaks for $(E \| y)$ move toward shorter wavelengths when the size of the holes and period are scaled down by the same coefficient, leaving the thickness of the layers the same. The shift is linearly proportional to the scaling factor.

We note that in the near-infrared spectral range, the experimentally measured transmission is lower than the calculated one by about a factor of two. This discrepancy is commonly observed and can be attributed to the roughness of the sample and the possible contamination occurring during the FIB milling process. ${ }^{9}$ Further discrepancies appear as a result of the beam focusing in our experiments. Such focusing leads to an effective integration of the measured transmission over a range of incident angles, determined by the numerical apertures of the microscope objectives.

In order to understand the origin of the main transmission resonance at $\sim 1.8 \mu \mathrm{m}$, we calculate the electric field distribution at three different planes within our sample. Figure 2 shows the combined picture of the electric field magnitude (color map) and direction (green lines) that enables us to visualize the current flow in the structure, since they are proportional to the electric field. From the images in Fig. 2 we come to two important conclusions: (i) most of the field is localized in the holes of the fishnet structure [see Fig. 2(a)]; and (ii) the current flow on the top and bottom metal plates is in the same direction [Figs. 2(b) and 2(c)]. The symmetry of the currents on the metal plates suggests that the observed resonance does not correspond to artificial magnetism as in Refs. 4, 8, and 17, but rather is related to the phenomena of extraordinary transmission in such fishnet metamaterials. $^{12}$

To further confirm this observation, in Fig. 3, we compare the transmission through the fishnet structure with the transmission through a thick gold metal film of $110 \mathrm{~nm}$, as well as the transmission through a bilayer $50 \mathrm{~nm}$ gold-30 nm titanium dioxide structure. Our simulations reveal similar transmission resonances for all the three structures suggesting negligible effects coming from the magnetic response in the trilayer fishnet structure.

\section{TILTED RESPONSE OF THE FISHNET METAMATERIAL}

Next, we determine the response of the metamaterial with respect to sample tilt in two orthogonal directions, for both polarizations of the incident electric field. We note that in general there are four cases of tilted excitation of the structure, as shown in the left column of Fig. 4, (i, ii) the trans- verse electric field component is along the thin wires $(E \| y)$ and the tilt is around the $x$ or $y$ axis [Figs. 4(a) and 4(b)]; and (iii, iv) the transverse electric field component is along the thicker metal strips $(E \| x)$ for tilts around $x$ or $y$ [Figs. 4(c) and $4(\mathrm{~d})$ ]. Commonly only two of these cases have been
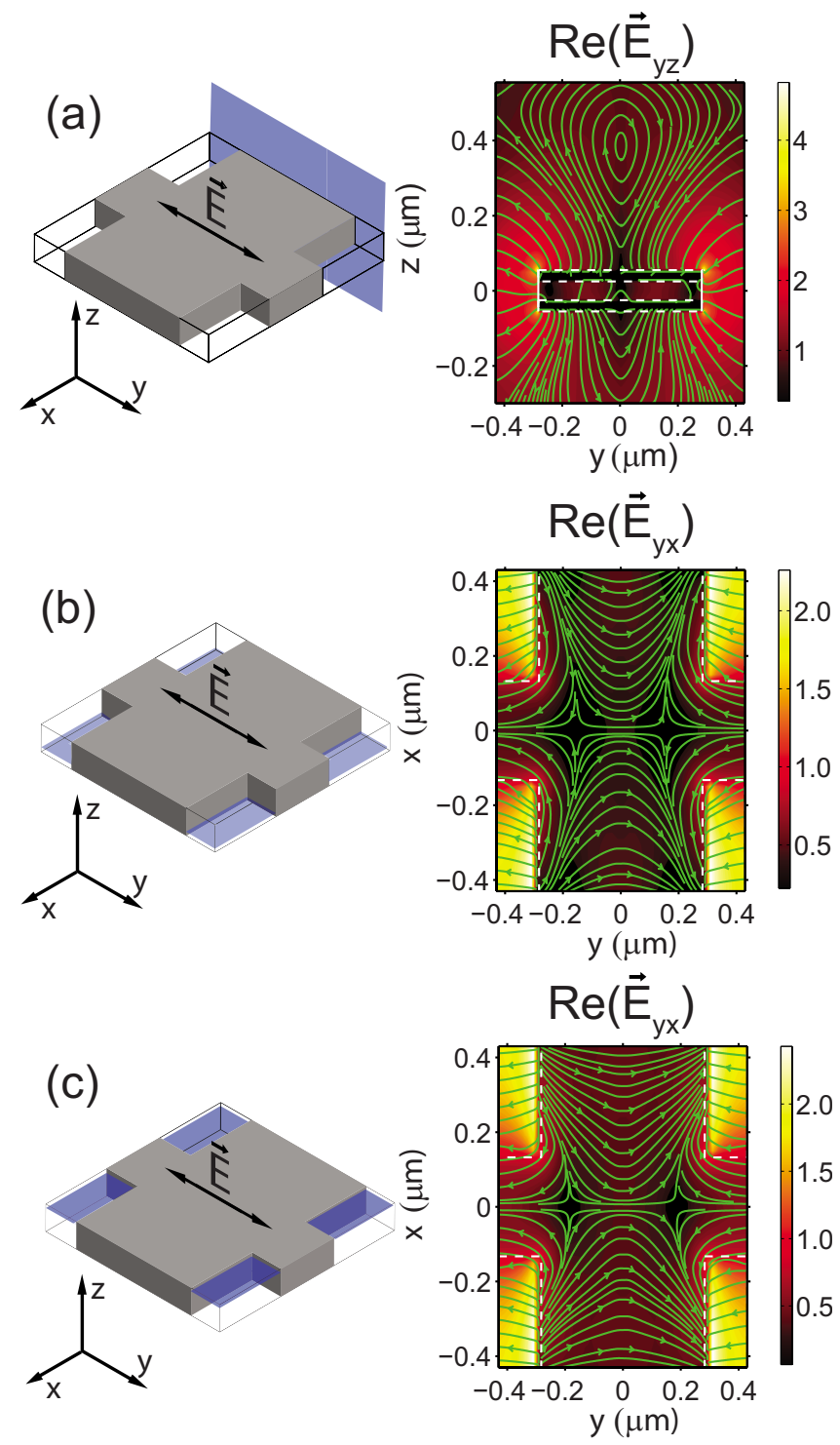

FIG. 2. (Color online) Numerically calculated electric field profiles for excitation at normal incidence and $\lambda=1.83 \mu \mathrm{m}$. Color scheme shows the magnitude of the electric field, while the green contours show its polarization. (a) Field cross-section through the middle of the hole in the $y-z$ plane. The substrate is on top of the structure for $z>0$. (b,c) Electric field distribution on the bottom and top metal films in the $x-y$ plane. 


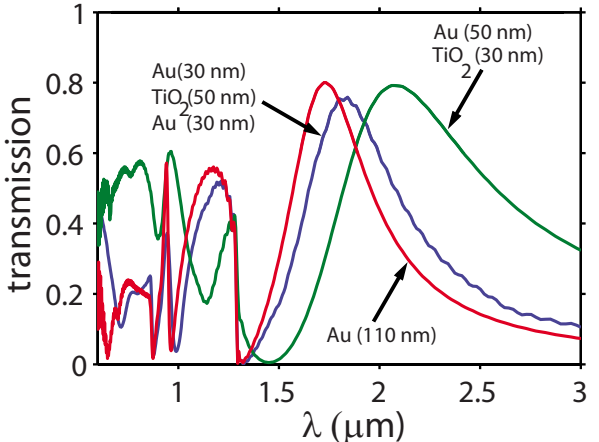

FIG. 3. (Color online) Comparison of the calculated spectra for normal incidence through our trilayer fishnet structure, two-layer $\mathrm{Au}(50 \mathrm{~nm})-\mathrm{TiO}_{2}(30 \mathrm{~nm})$, and pure gold of $110 \mathrm{~nm}$ thickness.

considered in the literature for a fabricated structure. For example in Ref. 4 only configurations (i) and (ii) have been investigated, while in Ref. 21 the analog of excitation (i) and (iii) have been discussed. This anisotropy is related to the dispersion of the fishnet polaritonic crystal, being different for both $x$ and $y$ direction. This is in contrast to the recently measured tilted response in symmetric Swiss-cross metamaterials that show no polarization anisotropy. ${ }^{22}$

In Fig. 4 (middle column) we show the numerically calculated transmission spectra as a function of sample tilt and polarization of the incident field. This transmission spectra are converted into dispersion diagrams using a similar approach as in the dispersion of single layer polaritonic crystals $^{23}$ and are shown in the right column of Fig. 4. In the case of incident polarization along the thin wires of the fishnet and rotation around the $x$ axis [Fig. 4(a)], we change the value of $k_{y}$ component of the wave vector as well as both the normal and tangential components of the incident electric field. On the transmission diagram one can see a hightransmission peak in the spectral region from 1.5 to $2.2 \mu \mathrm{m}$ which is associated with a wave guiding mode of the holes. This is clarified by independent calculations of the transmission spectra of a single hole of the fishnet and perfectly matching layer at the boundaries of the unit cell. The trans-
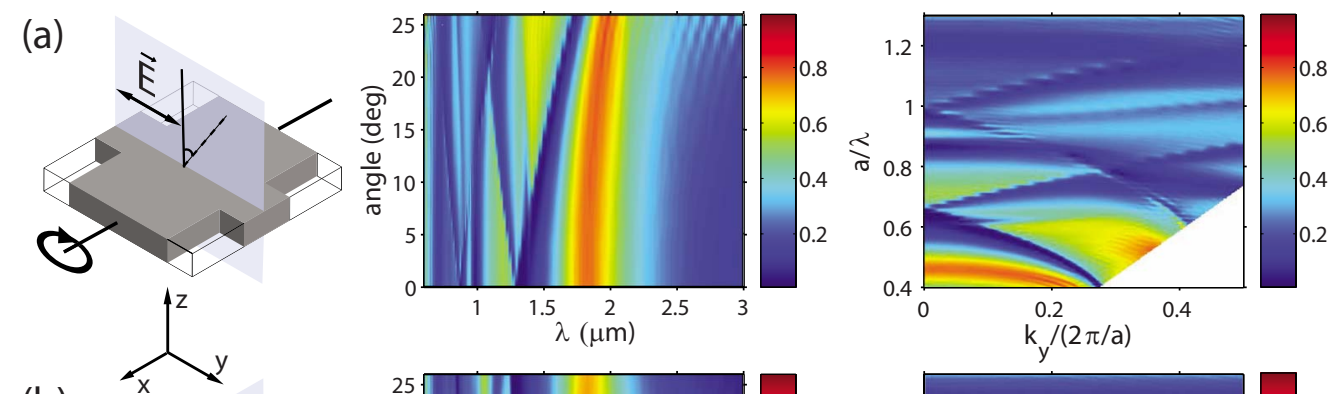

(b)
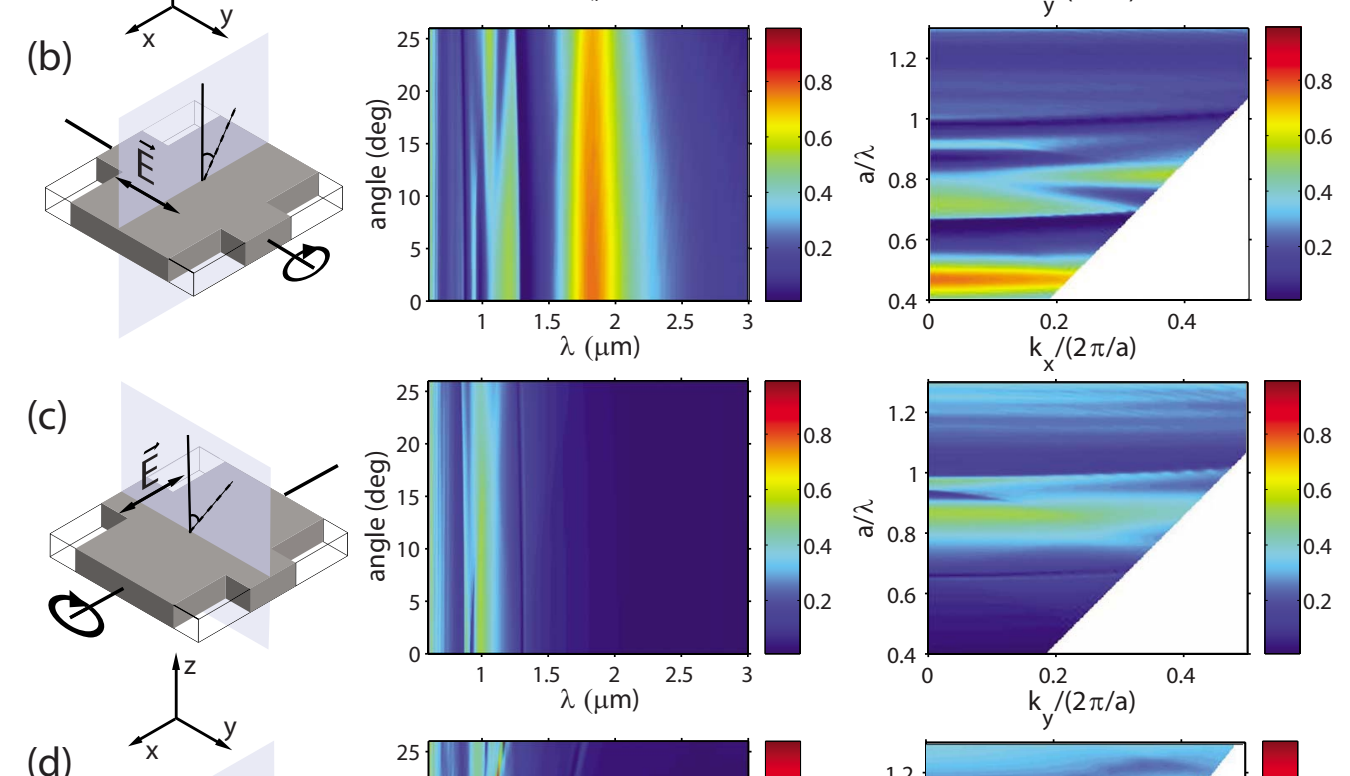

(d)
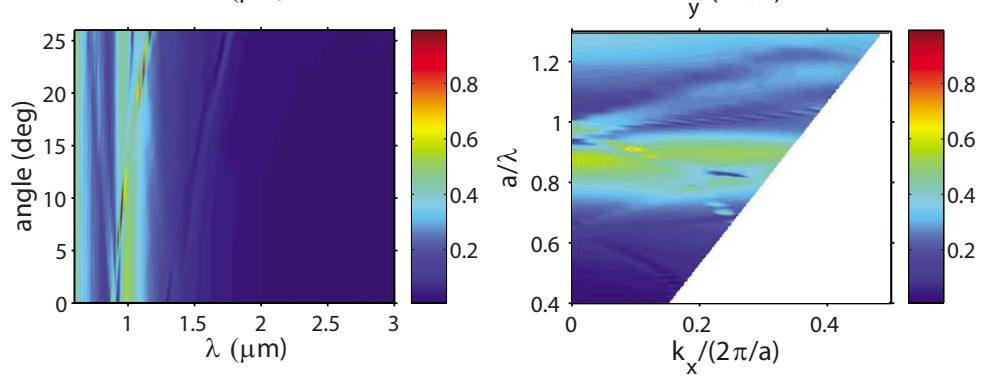

FIG. 4. (Color online) Calculated dispersion of our fishnet metamaterial. (Middle column) — transmission spectra vs. tilt of the sample in two orthogonal directions and excitation with $E_{y}$ and $E_{x}$ initial polarization (see left column). (Right column)—the corresponding dispersion diagrams. 

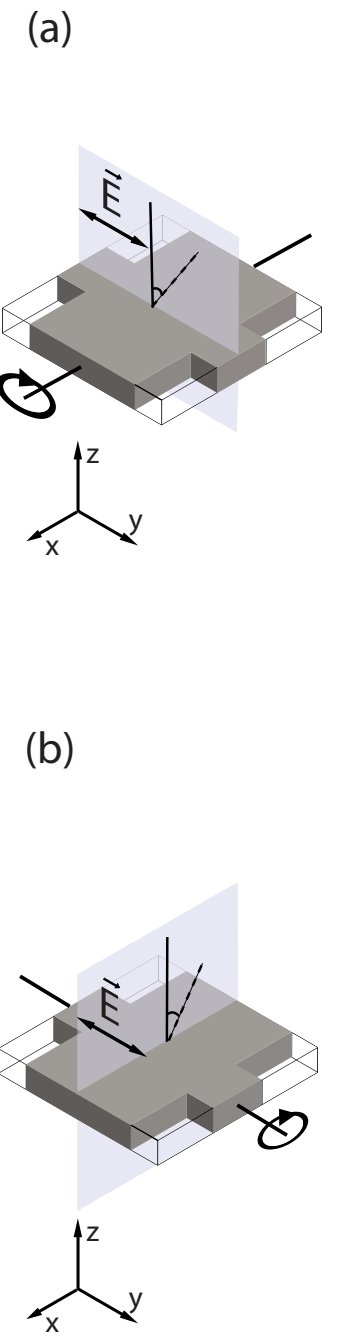
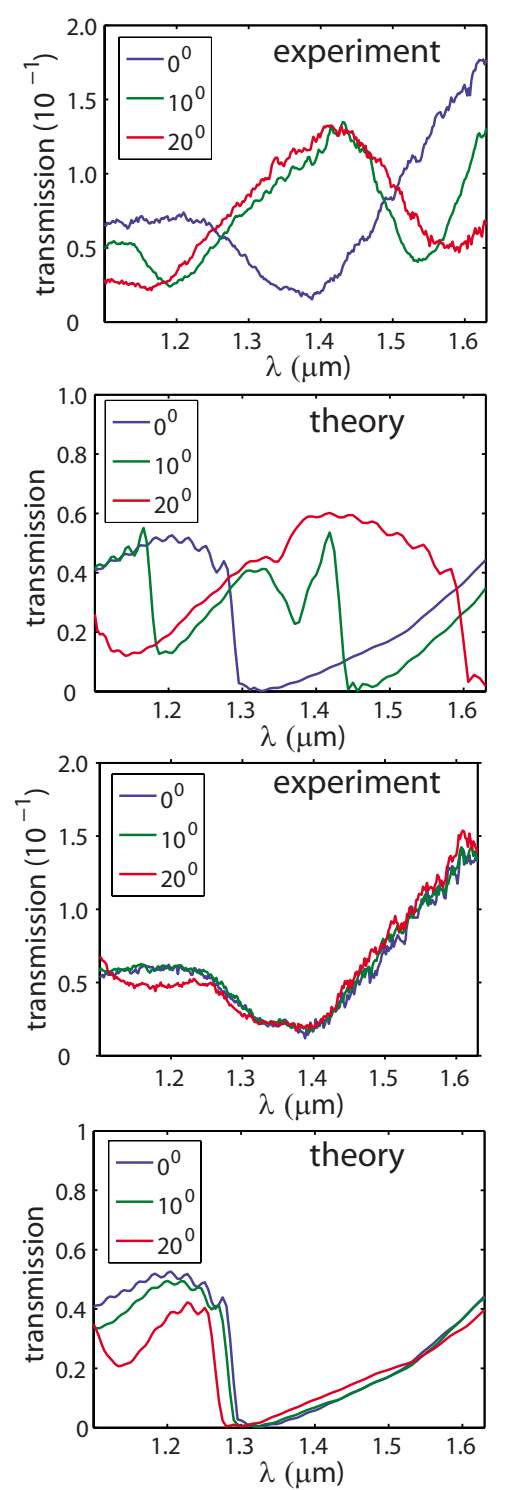

FIG. 5. (Color online) Comparison of the experimentally measured and numerically calculated transmission spectra for inclination angles of $0^{\circ}, 10^{\circ}$, and $20^{\circ}$ over the spectral range of the optical spectrum analyzer.

mission of a single hole showed a pronounced transmission maxima at $\sim 1.8 \mu \mathrm{m}$ matching well with the transmission peak shown in Fig. 1(d). The corresponding dispersion diagram (right column) also shows that the transmission dip at $1.3 \mu \mathrm{m}$ is connected with coupling to a surface plasmon with a field concentrated between the metal plates and on the top metal plate, extending into the substrate. This mode splits into two separate plasmon modes for oblique incidence and therefore its dispersion behavior brings significant changes to the spectrum as we vary the angle of incidence.

We tested this behavior experimentally by measuring the transmission spectra for three different tilt angles of the sample as shown in Fig. 5(a) top. For comparison in Fig. 5(a) bottom, we plot the numerical results [Fig. 4(a)] in the spectral range of our optical spectrum analyzer. The numerical simulations show good agreement with our experimental measurements, describing well the behavior of the spectral features, including the splitting of the transmission minima at $1.4 \mu \mathrm{m}$ at normal incidence into two separate plasmon coupling minima at $\sim 1.2$ and $1.5 \mu \mathrm{m}$ for oblique angles of incidence. There is a good agreement between theory and experiment for the wavelengths of the transmission dip, despite the overall lower transmission obtained experimentally.

With the same polarization but rotation around the thin wire ( $y$ axis) we vary the $x$ component of the $k$ vector, however keep a constant tangential component of the incident electric field [Fig. 4(b)]. In this case a surface plasmon should not demonstrate a dispersive behavior and, indeed, one could see that the transmission dip and spectra remain constant with the sample tilt. In the experiment we also observed no changes in the spectrum with a tilt of up to $20^{\circ}$ [Fig. 5(b)].

The stability of the spectrum suggests that this is the best arrangement of the fishnet pattern if one wants to work with tilted metamaterial surfaces, such as prisms ${ }^{9}$ or tilted slabs. ${ }^{19}$ If the fishnet is fabricated such that the thin wires are perpendicular to the slope of the tilted surface, surface plasmon dispersion should not significantly change the transmission spectrum for a wide range of incident angles. Such an arrangement is important since the sample tilt will not affect the effective parameters of the metamaterial and the observation of effects such as negative refraction will be unambiguous.

For the last two cases [Figs. 4(c) and 4(d)], when the polarization is perpendicular to the thin wires, we cannot observe regions with pronounced transmission resonances anymore. This is further proof that the high transmission is mostly governed by the hole modes of the structure while the periodicity, which is the same in both $x$ and $y$ directions, has much less influence.

\section{CONCLUSIONS}

We have fabricated and characterized $\mathrm{Au}-\mathrm{TiO}_{2}-\mathrm{Au}$ fishnet metamaterials operating at near-infrared wavelengths. We have demonstrated tuning of the transmission resonances when tilt is applied around an axis perpendicular to the plane of incident beam polarization. This tuning is due to the interplay between hole and surface plasmon modes in the fishnet structure. Remarkably stronger effects are observed when the rotation occurs over an axis coinciding with the wider metal strip of the fishnet structure. On the other hand, for rotation around the thin metal strips and incident polarization parallel to them, a robustness of the transmission resonances has been observed. Our results provide important direction for possible application of fishnet structures in beam steering applications, while preserving the spectral properties of the structure.

\section{ACKNOWLEDGMENTS}

The authors acknowledge funding from the Australian Research Council and from the National Computing Infrastructure Merit Allocation Scheme. We acknowledge useful discussions with N. Zheludev and A. Zayats. 
${ }^{1}$ V. M. Shalaev, Nat. Photonics 1, 41 (2007).

${ }^{2}$ R. A. Shelby, D. R. Smith, and S. Schultz, Science 292, 77 (2001).

${ }^{3}$ S. Zhang, W. Fan, N. C. Panoiu, K. J. Malloy, R. M. Osgood, and S. R. J. Brueck, Phys. Rev. Lett. 95, 137404 (2005).

${ }^{4}$ G. Dolling, M. Wegener, A. Schaedle, S. Burger, and S. Linden, Appl. Phys. Lett. 89, 231118 (2006).

${ }^{5}$ G. Dolling, C. Enkrich, M. Wegener, C. Soukoulis, and S. Linden, Science 312, 892 (2006).

${ }^{6}$ W. Wu, E. Kim, E. Ponizovskaya, Y. Liu, Z. Yu, N. Fang, Y. R. Shen, A. M. Bratkovsky, W. Tong, C. Sun, X. Zhang, S.-Y. Wang, and R. S. Williams, Appl. Phys. A: Mater. Sci. Process. 87, 143 (2007).

${ }^{7}$ U. K. Chettiar, A. V. Kildishev, H.-K. Yuan, W. Cai, S. Xiao, V. P. Drachev, and V. M. Shalaev, Opt. Lett. 32, 1671 (2007).

${ }^{8}$ T. Li, J.-Q. Li, F.-M. Wang, Q.-J. Wang, H. Liu, S.-N. Zhu, and Y.-Y. Zhu, Appl. Phys. Lett. 90, 251112 (2007).

${ }^{9}$ J. Valentine, S. Zhang, T. Zentgraf, E. Ulin-Avila, D. A. Genov, G. Bartal, and X. Zhang, Nature (London) 455, 376 (2008).

${ }^{10}$ E. Kim, F. Wang, W. Wu, Z. Yu, and Y. R. Shen, Phys. Rev. B 78, 113102 (2008).

${ }^{11}$ V. M. Shalaev, W. Cai, U. K. Chettiar, H.-K. Yuan, A. K. Sarychev, V. P. Drachev, and A. V. Kildishev, Opt. Lett. 30, 3356 (2005).

${ }^{12}$ A. Mary, S. G. Rodrigo, F. J. Garcia-Vidal, and L. Martin-
Moreno, Phys. Rev. Lett. 101, 103902 (2008).

${ }^{13}$ A. Mary, S. G. Rodrigo, L. Martin-Moreno, and F. J. GarciaVidal, Phys. Rev. B 80, 165431 (2009).

${ }^{14}$ C. Simovski, Metamaterials 2, 169 (2008).

${ }^{15}$ A. K. Sarychev, G. Shvets, and V. M. Shalaev, Phys. Rev. E 73, 036609 (2006).

${ }^{16}$ T. Li, S.-M. Wang, H. Liu, J.-Q. Li, F.-M. Wang, S.-N. Zhu, and X. Zhang, J. Appl. Phys. 103, 023104 (2008).

${ }^{17}$ H. Liu, Y. M. Liu, T. Li, S. M. Wang, S. N. Zhu, and X. Zhang, Phys. Status Solidi B 246, 1397 (2009).

${ }^{18}$ K. B. Alici and E. Ozbay, J. Phys. D 41, 135011 (2008).

${ }^{19}$ M. Beruete, M. Navarro-Cía, M. Sorolla, and I. Campillo, Phys. Rev. B 79, 195107 (2009).

${ }^{20}$ A. D. Rakic, A. B. Djurisic, J. M. Elazar, and M. L. Majewski, Appl. Opt. 37, 5271 (1998).

${ }^{21}$ T. F. Gundogdu, I. Tsiapa, A. Kostopoulos, G. Konstantinidis, N. Katsarakis, R. S. Penciu, M. Kafesaki, E. N. Economou, Th. Koschny, and C. M. Soukoulis, Appl. Phys. Lett. 89, 084103 (2006).

${ }^{22}$ C. Menzel, C. Helgert, J. Upping, C. Rockstuhl, E.-B. Kley, R. B. Wehrspohn, T. Pertsch, and F. Lederer, Appl. Phys. Lett. 95, 131104 (2009).

${ }^{23}$ W. Dickson, G. A. Wurtz, P. R. Evans, R. J. Pollard, and A. V. Zayats, Nano Lett. 8, 281 (2008). 